\title{
Some New Examples of Quasi-Invariant Measures on a Hilbert Space
}

By

\author{
Hiroaki SHImomura*
}

\begin{abstract}
Introduction
In this paper, we shall consider the translationally quasi-invariant measures on infinite-dimensional linear topological spaces, especially on a rigged Hilbert space $E \subset H \subset E^{*}$. Let $\mu$ be a measure on $E^{*}$. We say that a measure $\mu$ is quasi-invariant, if $\mu(A)=0$ implies $\mu(A+e)=0$ for all $e \in E^{*}$. After this definition, in the finite dimensional case we can characterize $\mu$ as the Lebesgue measure modulo equivalence of absolute continuity. But in the infinite-dimensional case this definition is unsuccessful, because there does not exist such measure except trivial one. On the other hand if we consider only those translations which are defined by the elements of $E$ or $H$, then there exist continuously many quasi-invariant measures which are singular with respect to each other. However the only explicit known example was the measure of Gaussian type up to the present time. But here we shall give two examples of translationally quasi-invariant ergodic measures which are essentially different from Gaussian ones. This is the purpose of the present paper. The author thanks to Professor H. Yoshizawa for the many valuable comments.
\end{abstract}

\section{$\S 1$. Preliminary Discussions}

Throughout this paper, all Hilbert spaces are real and separable. We follow the usual terminology in the infinite-dimensional measure

Communicated by H. Yoshizawa, November 11, 1974.

* Department of Mathematics, Fukui University, Fukui, Japan. 
theory. Let $E$ be a Hilbert space with the scalar product $<e, f\rangle_{E}$ and $H$ be another Hilbert space with the scalar product $\langle h, g\rangle_{H}$. Assume that $E$ is contained in $H$ as a dense subspace and the natural injection is a Hilbert-Shmidt operator. Let $E^{*}$ be the topological dual space of $E$. Then, identifying $H$ with $H^{*}$, we can assume $E \subset H \subset E^{*}$. We shall denote the value of $x \in E^{*}$ at $e \in E$ by $x(e)$.

For the most part of this paper, the discussion will be performed in this situation, so we shall state briefly the basic definitions and theorems with respect to the measure space on $E^{*}$. The general arguments are found in [1]. Let $\mu$ be a $\sigma$-finite measure on the Borel $\sigma$-field $\mathfrak{B}\left(E^{*}\right)$, which is generated by all open subsets in $E^{*}, \Phi$ be a linear subspace of $E^{*}$ and $\tau_{\varphi} \mu$ be a transformed measure of $\mu$ for $\varphi \in \Phi$.

$$
\tau_{\varphi} \mu(A)=\mu(A-\varphi) \quad \text { for all } \quad A \in \mathfrak{B}\left(E^{*}\right) \text {. }
$$

Definition 1.1. A measure $\mu$ on $\mathfrak{B}\left(E^{*}\right)$ is called $\Phi$-quasi-invariant, if and only if $\tau_{\varphi} \mu \cong \mu$ holds for all $\varphi \in \Phi$.

Here the relation $\cong$ means the equivalence relation of the absolute continuity, while $\mu_{1} \lesssim \mu_{2}$ means that $\mu_{1}$ is absolutely continuous with respect to $\mu_{2}$.

Definition 1.2. A measure $\mu$ on $\mathfrak{B}\left(E^{*}\right)$ is called strictly- $\Phi$-quasiinvariant, if the following condition is satisfied.

$$
\tau_{\varphi} \mu \cong \mu \quad \text { if and only if } \varphi \in \Phi \text {. }
$$

Definition 1.3. A measure $\mu$ on $\mathfrak{B}\left(E^{*}\right)$ is called $\Phi$-ergodic if the following two conditions are satisfied.

(1) $\mu$ is $\Phi$-quasi-invariant.

(2) For any $\Phi$-quasi-invariant measure $\mu^{\prime}$, the relation $\mu^{\prime} \lesssim \mu$ implies $\mu^{\prime}=0$ or $\mu^{\prime} \cong \mu$.

Theorem 101. Two 1.ergodic measures on $\mathfrak{B}\left(E^{*}\right)$ are equivalent or singular with each other.

Proof is omitted. See [1].

As any $\sigma$-finite measure is equivalent to a probability measure, we 
shall only consider a probability measure from now on. Let $e_{1}, e_{2}, \ldots$, $e_{n}, \ldots$ be elements in $E$, which are linearly independent and span the whole space $E$. Let $\pi_{n}$ be the mapping from $E^{*}$ to $R^{n}$ such that

$$
x \in E^{*} \longrightarrow\left(x\left(e_{1}\right), x\left(e_{2}\right), \ldots, x\left(e_{n}\right)\right) \in R^{n},
$$

and $\mu_{n}$ be the image measure of $\mu$ by the map $\pi_{n}$ for each $n$. Then we can state a simple criterion for equivalence in the following theorem.

Theorem 1.2. For absolute continuity of $v$ with respect to $\mu$, it is necessary and sufficient that the following two conditions are satisfied.

(1) $v_{n} \lesssim \mu_{n}$ holds as n-dimensional measures for each $n$.

(2) Put $\frac{d v_{n}}{d \mu_{n}}(y)=\rho_{n}(y)$ for $y \in R^{n}$, then $\left\{\sqrt{\rho_{n}\left(\pi_{n}(x)\right)}\right\}$ forms a Cauchy sequence in $L_{\mu}^{2}\left(E^{*}\right)$.

Proof is omitted. See [1].

\section{§2. Gaussian Measures}

Definition 2.1. A probability measurc $g$ on $\mathfrak{B}\left(E^{*}\right)$ is called Gaussian measure with mean vector $x_{0} \in E^{*}$ and variance operator $S$, if its Fourier-Bochner transformation

$$
\hat{g}(e)=\int_{E^{*}} \exp (i x(e)) d g(x)
$$

has the form $\hat{g}(e)=\exp \left(i x_{0}(e)-1 / 2\|\operatorname{Se}\|_{E}^{2}\right)$ for all $e \in E$, where $S$ is a positive definite Hilbert-Shmidt operator.

Remark 1. In order that $g$ is a $\sigma$-additive measure on $\mathfrak{B}\left(E^{*}\right)$, it is necessary and sufficient that $S$ is a Hilbert-Shmidt operator. This is an assertion of the Sazanov's theorem. See [2].

Remark 2. If $S$ is a degenerate operator, then $g$ is not an $E$ quasi-invariant measure.

Remark 3. If a Gaussian measure is E-quasi-invariant, then it 
is also an E-ergodic measure.

Theorem 2.1. Let $g$ be a Gaussian measure on $\mathfrak{B}\left(E^{*}\right)$ with mean vector $x_{0} \in E^{*}$ and variance operator $S$. Then in order that $g$ is a strictly-E-quasi-invariant measure, it is necessary and sufficient that there exist two positive constants $c_{1}$ and $c_{2}$ such that

$$
c_{1}\|e\|_{E^{*}} \leqq\|S e\|_{E} \leqq c_{2}\|e\|_{E^{*}} \quad \text { for all } e \in E .
$$

Further, under the above assumption, $g\left(H+x_{0}\right)=1$.

Proof. Without loss of generality, we can assume $x_{0}=0$. First of all we remark that for $y \in E^{*}, \tau_{y} g \simeq g$ holds, if and only if $y$ is continuous with respect to the semi-norm $\|S e\|_{E}$. This assertion can be found in [3]. Now we prove the necessity. If $g$ is strictly-E-quasi-invariant, then for any $x \in E$ there exists some $y \in E$ such that

$$
<y, S e>_{E}=<x, e>_{H} \quad \text { for all } e \in E
$$

by the above remark, and $y$ is unique for each $x$, as $S$ is a non degenerate operator. Thus we can define a map $T$ on $E, x \rightarrow y$, then $T$ is closed one to one and onto (in virtue of the strict-E-quasi-invariance), therefore the closed graph theorem assures that $T$ is a homeomorphic operator on $E$. Next we take a c.o.n.s. (complete orthonormal system) $e_{1}, e_{2}, \ldots, e_{n}, \ldots$ in $E$. Substituting $e_{j}$ for $x$ in (2), we get $<T e_{j}, S e e_{E}^{2}=$ $<e_{j}, e>_{H}^{2}$ and summing up over $j$, we get $\left\|T^{*} S e\right\|_{E}^{2}=\|e\|_{E^{*}}^{2}$ for all $e \in E$. As also $T^{*}$ is a homeomorphic operator on $E$, we get from the above equality,

$$
\|S e\|_{E} \cdot\left\|T^{*-1}\right\|^{-1} \leqq\|e\|_{E^{*}} \leqq\left\|T^{*}\right\| \cdot\|S e\|_{E} \cdot
$$

Conversely suppose that the inequality (1) is satisfied for $S$. We want to show that the functional $y \in E^{*}$ is continuous with respect to the norm $\|S e\|_{E}$, if and only if $y \in E$. But under the condition (1), for $y \in E^{*}$ the above continuity is equivalent with the continuity with respect to the norm $\|e\|_{E^{*}}$, therefore equivalent with the existence of such $z \in E^{*}$ as

$$
y(e)=<e, z>_{E^{* *}}
$$


Now let $e_{1}, e_{2}, \ldots, e_{n}, \ldots$ be a c.o.n.s. in $E$, whose elements are eigenvectors of the natural injection $E \rightarrow H$. Substituting $e_{j}$ for $e$ in (3), we get $y\left(e_{j}\right)=\left\|e_{j}\right\|_{H}^{2} z\left(e_{j}\right)$ in virtue of $\left\|e_{j}\right\|_{H}^{2}=\left\|e_{j}\right\|_{E^{*}}$. Therefore for an arbitrary element $e \in E$,

$$
\begin{aligned}
y(e) & =\sum_{j=1}^{\infty}<e, e_{j}>_{E} y\left(e_{j}\right)=\sum_{j=1}^{\infty}<e, e_{j}>_{E}\left\|e_{j}\right\|_{H}^{2} z\left(e_{j}\right) \\
& =\sum_{j=1}^{\infty}<e, e_{j}>_{H} z\left(e_{j}\right) .
\end{aligned}
$$

As $\sum_{j=1}^{\infty} z\left(e_{j}\right) e_{j}=\xi \in E$, and $y(e)=<e, \xi>_{H}, y$ is an element of $E$. Of course for every $\xi \in E,\langle e, \xi\rangle_{H}$ is continuous with respect to the norm $\|e\|_{E^{*}}$. Thus we have proved sufficiency. Lastly we shall consider its support. Here again we can assume $x_{0}=0$. Let $e_{1}, e_{2}, \ldots, e_{n}, \ldots$ have the same meaning in the proof of sufficiency. Then,

$$
\begin{aligned}
& H=\left\{x \in E^{*}: \sum_{j=1}^{\infty} x^{2}\left(e_{j}\right) /\left\|e_{j}\right\|_{H}^{2}<\infty\right\}, \quad \text { and } \\
& \begin{aligned}
g(H) & =\lim _{R \rightarrow \infty} \lim _{N \rightarrow \infty} g\left(x \in E^{*}: \sum_{j=1}^{N} x^{2}\left(e_{j}\right) /\left\|e_{j}\right\|_{H}^{2} \leqq R\right) \\
& =1-\lim _{R \rightarrow \infty} \lim _{N \rightarrow \infty} g\left(x \in E^{*}: \sum_{j=1}^{N} x^{2}\left(e_{j}\right) /\left\|e_{j}\right\|_{H}^{2}>R\right) \\
& \geqq 1-\lim _{R \rightarrow \infty} \lim _{N \rightarrow \infty} 1 / R \quad \sum_{j=1}^{N} \int_{E^{*}} x^{2}\left(e_{j}\right) /\left\|e_{j}\right\|_{H}^{2} d g(x) \\
& =1-\lim _{R \rightarrow \infty} \lim _{N \rightarrow \infty} 1 / R \quad \sum_{j=1}^{N}\left\|S e_{j}\right\|_{E}^{2} /\left\|e_{j}\right\|_{H}^{2} \\
& \geqq 1-\lim _{R \rightarrow \infty} \lim _{N \rightarrow \infty} c_{2}^{2} / R \quad \sum_{j=1}^{N}\left\|e_{j}\right\|_{H}^{2}=1 .
\end{aligned}
\end{aligned}
$$

Theorem 2.2. Under the same notation in Theorem 2.1, $g$ is strictly-H-quasi-invariant if and only if there exist two positive constants $c_{1}$ and $c_{2}$ such that

$$
c_{1}\|e\|_{H} \leqq\|S e\|_{E} \leqq c_{2}\|e\|_{H}
$$

Proof is carried out in a quite similar way, so we omit it.

\section{§3. Examples of Translationally Quasi-Invariant Measures}

In this section we shall give two examples of translationally E- 
quasi-invariant and $E$-ergodic measures on $\mathfrak{B}\left(E^{*}\right)$, which are singular with respect to every $E$-quasi-invariant Gaussian measure on $\mathfrak{B}\left(E^{*}\right)$ in the situation $E \subset H \subset E^{*}$. We assume that the natural injection $c$ is a Hilbert-Shmidt operator but it is not a nuclear operator. Let $e_{1}$, $e_{2}, \ldots, e_{n}, \ldots$ be a c.o.n.s. in $E$, whose elements are eigen-vectors of $\iota$ and put $h_{n}=e_{n} /\left\|e_{n}\right\|_{H}$ for each $n$. Then $h_{1}, h_{2}, \ldots, h_{n}, \ldots$ are mutually orthogonal in $H$. From now on we fix these symbols.

\section{Measure of Cauchy Type}

Let $\left\{\alpha_{j}\right\}$ be a square summable sequence of real positive numbers. We consider the following positive definite function defined on $E$.

$$
\hat{\lambda}(e)=\exp \left(-\sum_{j=1}^{\infty}\left|<e, e_{j}>_{E}\right| \alpha_{j}\right) .
$$

Then a unique distribution, which we shall denote by $\lambda$ corresponds to $\hat{\lambda}_{\text {. }}$

Lemma 3.1. In order that $\lambda$ is a $\sigma$-additive measure on $\mathfrak{B}\left(E^{*}\right)$, it is necessary and sufficient that $\left\{\alpha_{j}\right\}$ is summable.

Proof of sufficiency. Let $\left\{\alpha_{j}\right\} \in l^{1}$. Now if we consider this weak distribution on $E^{a}$ (algebraic dual of $E$ ), then it is necessarily $\sigma$-additive by the Kolmogorov's consistency theorem. We shall denote it by $\tilde{\lambda}_{\text {. }}$ Then as the $\sigma$-additiveness of $\lambda$ is equivalent to $\tilde{\lambda}\left(E^{*}\right)=1$, we shall estimate its value.

$$
\begin{gathered}
\tilde{\lambda}\left(E^{*}\right)=\tilde{\lambda}\left(x: \sum_{j=1}^{\infty} x^{2}\left(e_{j}\right)<\infty\right)=\lim _{R \rightarrow \infty} \lim _{N \rightarrow \infty} \lambda\left(x: \sum_{j=1}^{N} x^{2}\left(e_{j}\right) \leqq R^{2}\right) \\
=1-\lim _{R \rightarrow \infty} \lim _{N \rightarrow \infty} \lambda\left(x: \sum_{j=1}^{N} x^{2}\left(e_{j}\right)>R^{2}\right) . \\
\lambda\left(x: \sum_{j=1}^{N} x^{2}\left(e_{j}\right)>R^{2}\right) \leqq \frac{\sqrt{e}}{\sqrt{e}-1} \int_{E^{* *}}\left\{1-\exp \left(-1 / 2 R^{2} \sum_{j=1}^{N} x^{2}\left(e_{j}\right)\right)\right\} d \lambda(x) \\
\leqq \frac{\sqrt{e}}{\sqrt{e}-1} \int_{E^{*}} \int_{R^{N}}\left\{1-\exp \left(i / R \sum_{j=1}^{N} x\left(e_{j}\right) y_{j}\right)\right\} d \lambda(x) d g_{N}(y) \\
\leqq \frac{\sqrt{e}}{\sqrt{e}-1} \int_{R^{N}}\left\{1-\exp \left(-1 / R \sum_{j=1}^{N}\left|y_{j}\right| \alpha_{j}\right)\right\} d g_{N}(y)
\end{gathered}
$$




$$
\begin{aligned}
& \leqq \frac{\sqrt{e}}{\sqrt{e}-1}\left\{1-\prod_{j=1}^{N} \frac{1}{\sqrt{2 \pi}} \int_{-\infty}^{\infty} \exp \left(-t^{2} / 2\right) \exp \left(-|t| \alpha_{j} \mid R\right) d t\right\} \\
& \leqq \frac{\sqrt{e}}{\sqrt{e}-1}\left\{1-\prod_{j=1}^{N} \rho\left(\alpha_{j} / R\right)\right\} .
\end{aligned}
$$

Here we put,

$$
\rho(\alpha)=1 / \sqrt{2 \pi} \int_{-\infty}^{\infty} \exp \left(-t^{2} / 2\right) \exp (-|t| \alpha) d t
$$

Then, $1-\rho(\alpha)=1-2 / \sqrt{2 \pi} \exp \left(\alpha^{2} / 2\right) \int_{\alpha}^{\infty} \exp \left(-t^{2} / 2\right) d t=O(\alpha)(\alpha \rightarrow 0)$. As $\left\{\alpha_{j}\right\}$ $\in l^{1}$, we get $\sum_{j=1}^{\infty}\left(1-\rho\left(\alpha_{j}\right)\right)<\infty$, so the above product converges to a non zero number and tends to 1 as $R \rightarrow \infty$. This shows that $\tilde{\lambda}\left(E^{*}\right)=1$.

Proof of necessity. Let $\lambda$ be a $\sigma$-additive measure on $\mathfrak{B}\left(E^{*}\right)$. Then, $0<\int_{E^{*}} \exp \left(-1 / 2 \sum_{j=1}^{\infty} x^{2}\left(e_{j}\right)\right) d \lambda(x)=\lim _{N \rightarrow \infty} \int_{E^{*}} \exp \left(-1 / 2 \quad \sum_{j=1}^{N} x^{2}\left(e_{j}\right)\right) d \lambda(x)=$ $\lim _{N \rightarrow \infty} \prod_{j=1}^{N} \rho\left(\alpha_{j}\right)$, where $\rho(\alpha)$ is defined in (4). Therefore, it is necessary that

$$
\sum_{j=1}^{\infty}\left(1-\rho\left(\alpha_{j}\right)\right)<\infty
$$

Since $1-\rho(\alpha)=O(\alpha)(\alpha \rightarrow 0)$, from (5) we conclude $\sum_{j=1}^{\infty} \alpha_{j}<\infty$. Q.E.D.

Remark 1. For $\left\{\alpha_{j}\right\} \in l^{2}$, if we set $p(e)=\sum_{j=1}^{\infty}\left|<e, e_{j}\right\rangle_{E} \mid \alpha_{j}$, then $p$ is a norm on $E$ and $\sum_{j=1}^{\infty} p^{2}\left(e_{j}\right)<\infty$. This shows $\hat{\lambda}(e)$ is continuous with respect to a Hilbert-Shmidt norm. But $p$ is not Hilbertian norm. So we cannot apply the Sazanov's theorem. This remark is due to professor Yamasaki.

Now we call these measures $\lambda\left(\sum \alpha_{j}<\infty\right)$ Cauchy type, as their onedimensional projection measures are Cauchy measures.

Lemma 3.2. Under the same notation in Lemma 3.1, $\lambda(H)=1$ is equivalent to $\sum_{j=1}^{\infty} \alpha_{j} /\left\|e_{j}\right\|_{H}<\infty$.

Proof. The assertion can be proved in a quite similar way, so we omit it. 
Lemma 3.3. Let $\eta \in E^{*}, \eta_{j}=\eta\left(e_{j}\right)$ for each $e_{j} \in E$, and $\lambda$ be a measure of Cauchy type with $\sum_{j=1}^{\infty} \alpha_{j}<\infty$. Then $\tau_{\eta} \lambda \simeq \lambda$ holds if and only if $\sum_{j=1}^{\infty} \eta_{j}^{2} / \alpha_{j}^{2}<\infty$.

Proof. We shall investigate the condition of Theorem 1.2. Let $\pi_{n}$ be a map from $E^{*}$ to $R^{n}$ such that $\pi_{n}(x)=\left(x\left(e_{1}\right), \ldots, x\left(e_{n}\right)\right)$ and $\lambda_{n}\left(\lambda_{n}^{\eta}\right)$ be the image measure of $\lambda\left(\tau_{\eta} \lambda\right)$ by the map $\pi_{n}$. Then, for $y=\left(y_{1}\right.$, $\left.y_{2}, \ldots, y_{n}\right) \in R^{n}$

$$
\begin{aligned}
\hat{\lambda}_{n}(y) & =\int_{R^{n}} \exp \left(i \sum_{j=1}^{n} y_{j} x_{j}\right) d \lambda_{n}(x)=\exp \left(-\sum_{j=1}^{n}\left|y_{j}\right| \alpha_{j}\right) \\
& =\pi^{-n} \alpha_{1} \cdots \alpha_{n} \int_{R^{n}} \prod_{j=1}^{n}\left(x_{j}^{2}+\alpha_{j}^{2}\right)^{-1} \exp \left(i \sum_{j=1}^{n} y_{j} x_{j}\right) d x_{1} d x_{2} \cdots d x_{n} .
\end{aligned}
$$

Therefore, $d \lambda_{n}(x)=\frac{\alpha_{1} \cdots \alpha_{n}}{\pi^{n}} \frac{1}{\prod_{j=1}^{n}\left(x_{j}^{2}+\alpha_{j}^{2}\right)} d x_{1} d x_{2} \cdots d x_{n}$. In the same way,

$$
d \lambda_{n}^{n}(x)=\frac{\alpha_{1} \cdots \alpha_{n}}{\pi^{n}} \frac{1}{\prod_{j=1}^{n}\left(\left(x_{j}-\eta_{j}\right)^{2}+\alpha_{j}^{2}\right)} d x_{1} d x_{2} \cdots d x_{n}
$$

Thus

$$
\frac{d \lambda_{n}^{\eta}}{d \lambda_{n}}(x)=\frac{\prod_{j=1}^{n}\left(x_{j}^{2}+\alpha_{j}^{2}\right)}{\prod_{j=1}^{n}\left(\left(x_{j}-\eta_{j}\right)^{2}+\alpha_{j}^{2}\right)}
$$

We put

$$
\sqrt{\frac{d \lambda_{n}^{\eta}}{d \lambda_{n}}}(x)=\sigma_{n}(x)
$$

Then,

$$
\begin{aligned}
& \int_{E^{*}}\left|\sigma_{n}\left(\pi_{n}(x)\right)-\sigma_{m}\left(\pi_{m}(x)\right)\right|^{2} d \lambda(x) \\
= & 2-2 \int_{E^{*}} \sigma_{n}\left(\pi_{n}(x)\right) \cdot \sigma_{m}\left(\pi_{m}(x)\right) d \lambda(x) \\
= & 2-\frac{2}{\pi^{n-m}} \int_{R} n-m \prod_{j=m+1}^{n} \frac{\alpha_{j}}{\sqrt{\left(x_{j}-\eta_{j}\right)^{2}+\alpha_{j}^{2}} \sqrt{x_{j}^{2}+\alpha_{j}^{2}}} d x_{m+1} \cdots d x_{n} \\
= & 2\left\{1-\prod_{j=m+1}^{n} \frac{1}{\pi} \int_{-\infty}^{\infty} \frac{d t}{\sqrt{\left(t-w_{j}\right)^{2}+1} \sqrt{t^{2}+1}}\right\}=2\left\{1-\prod_{j=m+1}^{n} \rho\left(w_{j}\right)\right\} .
\end{aligned}
$$


Here we put

$$
\rho(w)=\frac{1}{\pi} \int_{-\infty}^{\infty} \frac{d t}{\sqrt{(t-w)^{2}+1} \sqrt{t^{2}+1}} \text { and } w_{j}=\frac{\eta_{j}}{\alpha_{j}} .
$$

Then in order that $\left\{\sigma_{n}\left(\pi_{n}(x)\right)\right\}$ forms a Cauchy sequence, it is necessary and sufficient that $\prod_{j=1}^{\infty} \rho\left(w_{j}\right)$ converges to a non zero number, which is equivalent to $\sum_{j=1}^{\infty}\left(1-\rho\left(w_{j}\right)\right)<\infty$. Therefore we have only to estimate its order at 0 . After some calculations, we get $\rho(w)=1+1 / 2 \rho^{\prime \prime}(\theta w) w^{2}$, $\rho^{\prime \prime}(0)=-1 / 8(0<\theta<1)$ and $\rho^{\prime \prime}(w)$ is a continuous function of $w$. Thus, $1-\rho(w)=O\left(w^{2}\right)(w \rightarrow 0)$. From this we get a desired conclusion.

Q.E.D.

From now on we put $\alpha_{j}=\left\|e_{j}\right\|_{H}^{2}$ for all $j$. Then the measure $\lambda_{0}$ corresponding to $\left\{\left\|e_{j}\right\|_{H}^{2}\right\}$ has the following properties in virtue of the preceding Lemmas.

(1) $\lambda_{0}$ is a $\sigma$-additive probability measure on $\mathfrak{B}\left(E^{*}\right)$ and $\lambda_{0}(H)$ $=0$.

(2) $\lambda_{0}$ is a strictly-E-quasi-invariant measure.

For, $\tau_{\eta} \lambda_{0} \cong \lambda_{0}$ holds if and only if $\sum_{j=1}^{\infty} \eta^{2}\left(e_{j}\right) /\left\|e_{j}\right\|_{H}^{4}<\infty$, in virtue of Lemma 3.3. This shows firstly $\sum_{j=1}^{\infty} \eta^{2}\left(h_{j}\right)<\infty$, so putting $\xi=\sum_{j=1}^{\infty} \eta\left(h_{j}\right) h_{j}$ $\in H$, we have $\eta(e)=\langle e \xi\rangle_{H}$ for all $e \in E$. And secondly, $\eta\left(e_{j}\right)=\eta\left(h_{j}\right)\left\|e_{j}\right\|_{H}$ shows $\sum_{j=1}^{\infty} \eta\left(h_{j}\right)^{2} /\left\|e_{j}\right\|_{H}^{2}<\infty$. Therefore,

$$
\xi=\sum_{j=1}^{\infty} \eta\left(h_{j}\right) /\left\|e_{j}\right\|_{H} e_{j} \in E .
$$

Conversely, if $\eta \in E, \eta=\sum_{j=1}^{\infty} \eta_{j} e_{j}$ with $\left\{\eta_{j}\right\} \in l^{2}$, then $\eta\left(e_{j}\right)=\eta_{j}\left\|e_{j}\right\|_{H}^{2}$ for each $j$, and

$$
\sum_{j=1}^{\infty} \eta^{2}\left(e_{j}\right) /\left\|e_{j}\right\|_{H}^{4}=\sum_{j=1}^{\infty} \eta_{j}^{2}<\infty
$$

Lemma 3.4. $\lambda_{0}$ is an E-ergodic measure.

Proof. For the proof of ergodicity, we refer the following proposition.

$(\mathbb{P})^{*}$ Let $y=y_{1} e_{1}+\cdots+y_{n} e_{n} \in E$. Then in order that $\lambda_{0}$ is E-ergodic, it is sufficient that thcre exists Borel function $f_{n}$ for each $n$ such that 


$$
\frac{d \tau_{y} \lambda_{0}}{d \lambda_{0}}(x)=f_{n}\left(x\left(e_{1}\right), \ldots, x\left(e_{n}\right)\right)
$$

In the present case this condition is assured by (6) in Lemma 3.3.

Q.E.D.

Lemma 3.5. For every $y \in E^{*}, \lambda_{0}(H+y)=0$ 。

Proof. We put $y_{j}=y\left(h_{j}\right)$. Then,

$$
\begin{aligned}
& \lambda_{0}(H+y)=\lim _{R \rightarrow \infty} \lim _{N \rightarrow \infty} \lambda_{0}\left(x: \sum_{j=1}^{N}\left(x\left(h_{j}\right)-y_{j}\right)^{2} \leqq 2 R^{2}\right) \\
& \quad \leqq e \lim _{R \rightarrow \infty} \lim _{N \rightarrow \infty} \int_{E^{*}} \exp \left(-1 / 2 R^{2} \sum_{j=1}^{N}\left(x\left(h_{j}\right)-y_{j}\right)^{2}\right) d \lambda_{0}(x) \\
& \quad \leqq e \lim _{R \rightarrow \infty} \lim _{N \rightarrow \infty} \int_{E^{*}} \int_{R^{N}} \exp \left(i / R \sum_{j=1}^{N}\left(x\left(h_{j}\right)-y_{j}\right) z_{j}\right) d \lambda_{0}(x) d g_{N}(z) \\
& \leqq e \lim _{R \rightarrow \infty} \lim _{N \rightarrow \infty} \int_{R^{N}} \exp \left(i \sum_{j=1}^{N} y_{j} z_{j} / R\right) \exp \left(-1 / R \sum_{j=1}^{N}\left\|e_{j}\right\|_{H}\left|z_{j}\right|\right) d g_{N}(z) \\
& \leqq e \lim _{R \rightarrow \infty} \lim _{N \rightarrow \infty} \prod_{j=1}^{N} \int 1 / \sqrt{2 \pi} \exp \left(i t y_{j} / R\right) \exp \left(-\left\|e_{j}\right\|_{H}|t| / R\right) \exp \left(-t^{2} / 2\right) d t \\
& \leqq e \lim _{R \rightarrow \infty} \lim _{N \rightarrow \infty} \prod_{j=1}^{N} \rho_{j} .
\end{aligned}
$$

Here we put

$$
\rho_{j}=1 / \sqrt{2 \pi} \int_{-\infty}^{\infty} \exp \left(i y_{j} t / R\right) \exp \left(-\left\|e_{j}\right\|_{H}|t| / R\right) \exp \left(-t^{2} / 2\right) d t
$$

Then $\rho_{j} \leqq \rho\left(\left\|e_{j}\right\|_{H} / R\right)$ for each $j$ and $\prod_{j=1}^{\infty} \rho\left(\left\|e_{j}\right\|_{H} / R\right)=0$ from the order of $\rho(\alpha)$ at 0 .

Q.E.D.

Theorem 3.1. Let $\lambda_{0}$ be the probability measure on $\mathfrak{B}\left(E^{*}\right)$, whose Fourier-Bochner transformation is

$$
\hat{\lambda}_{0}(e)=\exp \left(-\sum_{j=1}^{\infty}\left|<e, e_{j}>_{E}\right|\left\|e_{j}\right\|_{H}^{2}\right)
$$

Then,

(1) $\lambda_{0}$ is a strictly-E-quasi-invariant and E-ergodic measure.

\footnotetext{
* This proposition is found as a note in [1].
} 
(2) $\lambda_{0}$ is singular with respect to every E-quasi-invariant Gaussian measure.

Proof. The first part was proved by the preceding several Lemmas. As for the second part, if $\lambda_{0}$ is not singular with respect to some $E$ quasi-invariant Gaussian measure $g$ with mean vector $x_{0} \in E^{*}$ and variance operator $S$, then by Theorem 1.1 and by Remark 3 after Definition 2.1, $g$ must be equivalent with $\lambda_{0}$. So $g$ is a strictly- $E$-quasi-invariant measure. Thus, $g\left(H+x_{0}\right)=1$ in virtue of Theorem 2.1. But on the other hand Lemma 3.5 shows that $\lambda_{0}\left(H+x_{0}\right)=0$. Therefore $\lambda_{0}$ must be singular with respect to $g$.

Q.E.D.

\section{Measure of Laplace Type}

In the preceding example, we used the fact that

$$
1 / \pi \int_{-\infty}^{\infty} e^{i s t} \frac{d s}{1+s^{2}}=\exp (-|s|)
$$

But herein we use the converse relation,

$$
1 / 2 \int_{-\infty}^{\infty} e^{i s t} \exp (-|s|) d s=\left(1+t^{2}\right)^{-1}
$$

We consider the following positive definite function on $E$,

$$
\hat{v}(e)=\prod_{j=1}^{\infty}\left(1+<e, e_{j}>_{E}^{2} \alpha_{j}^{2}\right)^{-1}
$$

where $\left\{\alpha_{j}\right\} \in l^{2}$, whose components are real positive numbers. In this case, the corresponding weak distribution $v$ is a $\sigma$-additive probability measure on $\mathfrak{B}\left(E^{*}\right)$. Further, $v(H)=1$ is equivalent to $\sum_{j=1}^{\infty} \alpha_{j}^{2} /\left\|e_{j}\right\|_{H}^{2}<\infty$. These assertions are shown in the same manner as before.

Lemma 3.6. Let $\eta \in E^{*}$. Then $\tau_{\eta} v \cong v$ holds, if and only if $\sum_{j=1}^{\infty} \eta^{2}\left(e_{j}\right) / \alpha_{j}^{2}<\infty$

Proof. As before, let $\pi_{n}$ be the map from $E^{*}$ to $R^{n}$ such that $\pi_{n}(x)=\left(x\left(e_{1}\right), x\left(e_{2}\right), \ldots, x\left(e_{n}\right)\right)$, and $v_{n}\left(v_{n}^{\eta}\right)$ be the image measure of $v$ $\left(\tau_{\eta} v\right)$ by the map $\pi_{n}$, respectively. Then for $y=\left(y_{1}, y_{2}, \ldots, y_{n}\right) \in R^{n}$, 


$$
\begin{aligned}
\hat{v}_{n}(y) & =\int_{E^{*}} e^{i\left(x_{1} y_{1}+\cdots+x_{n} y_{n}\right)} d v_{n}(x) \\
& =\prod_{j=1}^{n} \frac{1}{1+\alpha_{j}^{2} y_{j}^{2}} \\
& =\frac{1}{2^{n} \alpha_{1} \cdots \alpha_{n}} \int_{R^{n}} e^{i\left(x_{1} y_{1}+\cdots+x_{n} y_{n}\right)} \exp \left(-\sum_{j=1}^{n} \frac{\left|x_{j}\right|}{\alpha_{j}}\right) d x_{1} \cdots d x_{n} .
\end{aligned}
$$

Therefore,

$$
d v_{n}(x)=\frac{1}{2^{n} \alpha_{1} \cdots \alpha_{n}} \exp \left(-\sum_{j=1}^{n} \frac{\left|x_{j}\right|}{\alpha_{j}}\right) d x_{1} d x_{2} \cdots d x_{n} .
$$

In the same way,

$$
d v_{n}^{\prime \prime}(x)=\frac{1}{2^{n} \alpha_{1} \cdots \alpha_{n}} \exp \left(-\sum_{j=1}^{n} \frac{\left|x_{j}-\eta_{j}\right|}{\alpha_{j}}\right) d x_{1} d x_{2} \cdots d x_{n},
$$

where we put $\eta_{j}=\eta\left(e_{j}\right)$. Thus,

$$
\frac{d v_{n}^{n}}{d v_{n}}(x)=\exp \left(-\sum_{j=1}^{n} \frac{1}{\alpha_{j}}\left\{\left|x_{j}-\eta_{j}\right|-\left|x_{j}\right|\right\}\right) \text { for } x \in R^{n}
$$

Now let

$$
\sigma_{n}(x)=\sqrt{\frac{d v_{n}^{\eta}}{d v_{n}}}(x)
$$

and we shall investigate the conditions of Theorem 1.2.

$$
\begin{aligned}
& \int_{E^{*}} \sigma_{n}\left(\pi_{n}(x)\right) \sigma_{m}\left(\pi_{m}(x)\right) d v(x) \\
& \quad=\prod_{j=m+1}^{n} \frac{1}{2 \alpha_{j}} \int_{-\infty}^{\infty} \exp \left(-\frac{1}{2 \alpha_{j}}\left\{\left|t-\eta_{j}\right|+|t|\right\}\right) d t=\prod_{j=m+1}^{n} \rho_{j}
\end{aligned}
$$

Here we put

$$
\rho_{j}=\frac{1}{2 \alpha_{j}} \int_{-\infty}^{\infty} \exp \left(-\frac{1}{2 \alpha_{j}}\left\{\left|t-\eta_{j}\right|+|t|\right\}\right) d t
$$

Then,

$$
\rho_{j}=1 / 2 \int_{-\infty}^{\infty} \exp \left(-1 / 2\left\{\left|t-w_{j}\right|+|t|\right\}\right) d t=1 / 2 e^{-\left|w_{j}\right| / 2}\left(2+\left|w_{j}\right|\right)
$$


and $1-\rho_{j}=O\left(w_{j}^{2}\right)\left(w_{j} \rightarrow 0\right)$, where $w_{j}=\eta_{j} / \alpha_{j}$. From the above arguments we conclude that, in order that $\left\{\sigma_{n}\left(\pi_{n}(x)\right)\right\}$ be a Cauchy sequence in $L_{v}^{2}\left(E^{*}\right)$, it is necessary and sufficient that $\left\{\eta_{j} / \alpha_{j}\right\} \in l^{2}$.

Q.E.D.

From now on, we put $\alpha_{j}=\left\|e_{j}\right\|_{H}$ for each $j$ and denote the corresponding measure by $v_{0}$. We call it Laplace type. Then $v_{0}$ has the following properties.

\section{Theorem 3.2.}

(1) $v_{0}$ is a $\sigma$-additive probability measure on $\mathfrak{B}\left(E^{*}\right)$, and $v_{0}(H)=0$.

(2) $v_{0}$ is a strictly-H-quasi-invariant and E-ergodic measure.

(3) $\int_{E^{*}} x(e) x(f) d v_{0}(x)=2<e, f>_{H}$, for all $e$ and $f \in E$.

(4) $v_{0}$ is singular with respect to every E-quasi-invariant Gaussian measure.

Proof. (1) is assured by the arguments in the first part of this paragraph. For (2), we have shown that in the last Lemma, $\tau_{\eta} v_{0} \cong v_{0}$ holds if and only if

$$
\sum_{j=1}^{\infty} \eta^{2}\left(e_{j}\right) /\left\|e_{j}\right\|_{I I}^{2}<\infty
$$

which is equivalent to $\eta \in H$. The assertion of ergodicity can bc proved by the proposition $(P)$ in Lemma 3.4 and (7) in Lemma 3.6. For (3),

$$
\begin{aligned}
& \int_{E^{*}} x^{2}\left(e_{j}\right) d v_{0}(x)=\frac{1}{2\left\|e_{j}\right\|_{H}} \int_{-\infty}^{\infty} \exp \left(-|x| /\left\|e_{j}\right\|_{H}\right) x^{2} d x \\
& =1 / 2\left\|e_{j}\right\|_{H}^{2} \int_{-\infty}^{\infty} \exp (-|x|) x^{2} d x=2\left\|e_{j}\right\|_{H}^{2}
\end{aligned}
$$

holds for each $j$. As $x\left(e_{1}\right), x\left(e_{2}\right), \ldots, x\left(e_{n}\right), \ldots$ are stochastic independent random variables for $v_{0}$, for arbitrary real numbers $c_{1}, c_{2}, \ldots$

$$
\int_{E^{*}} x^{2}\left(\sum_{j=1}^{\infty} c_{j} e_{j}\right) d v_{0}(x)=2 \sum_{j=1}^{n}\left\|c_{j} e_{j}\right\|_{H}^{2}
$$

Now we can extend the above equality to an arbitrary element $e \in E$ by the continuity of both sides. From this we can easily derive the requested result. Lastly we shall prove (4). But before it, we state a 
lemma.

Lemma 3.7. Let $A_{j}=\left\{x:\left|x\left(h_{j}\right)\right| \geqq \log j\right\}$ for each $j$. Then for any $x_{0} \in E^{*}, v_{0}\left(\varlimsup_{j} A_{j}+x_{0}\right)=1$, while $g\left(\varlimsup_{j} A_{j}\right)=0$ for every strictly-Hquasi-invariant Gaussian measure $g$ with mean vector 0 and variance operator $S$.

Proof. $\quad v_{0}\left(A_{j}+x_{0}\right)=1 / 2 \int_{\left|t-x_{0}\left(h_{j}\right)\right| \geqq \log j} e^{-|t|} d t \geqq 1 / 2 j \quad$ is an easy calculation. As $\left\{A_{j}+x_{0}\right\}(j=1,2, \ldots)$ is a stochastic independent sequence of sets for $v_{0}, v_{0}\left(\varlimsup_{j}\left(A_{j}+x_{0}\right)\right)=1$ holds by the Borel-Cantelli's lemma. As for $g, S$ must satisfy $c_{1}\|e\|_{H} \leqq\|S e\|_{E} \leqq c_{2}\|e\|_{H}$ for all $e \in E$, in virtue of Theorem 2.1. Now,

$$
\begin{aligned}
g\left(A_{j}\right) & =\frac{1}{\sqrt{2 \pi}\left\|S h_{j}\right\|_{E}} \int_{|x| \geqq \log j} \exp \left(-\frac{x^{2}}{2\left\|S h_{j}\right\|_{E}^{2}}\right) d x=\frac{2}{\sqrt{2 \pi}} \int_{\frac{\log j}{\left\|S h_{j}\right\|_{E}}}^{\infty} e^{-x^{2} / 2} d x \\
& =o\left(j^{-2}\right) \quad(j \rightarrow \infty) .
\end{aligned}
$$

In the last step we used the relation $c_{1} \leqq\left\|S h_{j}\right\|_{E} \leqq c_{2}$. Again using the Borel-Cantelli's lemma, we get $g\left(\varlimsup_{j} A_{j}\right)=0$ 。 Q.E.D.

Returning to the proof of (4), if $v_{0}$ is not singular with respect to an $E$-quasi-invariant Gaussian measure $g$ with mean vector $x_{0} \in E^{*}$ and variance operator $S$, then $v_{0}$ and $g$ must be equivalent with each other as before. Therefore $g$ is a strictly- $H$-quasi-invariant measure. But Lemma 3.7 shows that there exists a set $A$ such that $g\left(A+x_{0}\right)=0$, while $v_{0}\left(A+x_{0}\right)=1$. This is a contradiction.

Q.E.D.

\section{References}

[1] Umemura, Y., Measures on infinite dimensional vector spaces, Publ. RIMS. Kyoto Univ. 1 (1965), 1-47.

[2] Prohorov, Yu. V., The method of characteristic functionals, Proceedings of the Fourth Berkley Symposium on Mathematical Statistics and Probability, Berkley and Los Angeles, University of California Press, 2 (1961), 403-419.

[3] Dudley, R. M., Singular translates of measures on linear spaces, Zeitshrift für Wahrscheinlichkeitstheorie, 3 (1964), 128-137.

[4] Gelfand, I. M. and Vilenkin, N.Ya., Generalized functions, $\mathbb{1}$ (English trans, Academic Press), 1961. 
[5] Parthasarathy, K. R., Probability measures on metric spaces, Academic Press, 1967.

[6] Feldman, J., Equivalence and perpendicularity of Gaussian processes, Pacific J. Math., 8 (1958), 128-137. 
